\title{
Relapse pattern and prognostic factors for patients with primary central nervous system lymphoma
}

\author{
Jeong Eun Kim ${ }^{1}$, Dok Hyun Yoon ${ }^{1}$, Shin $\mathrm{Kim}^{1}$, Dae Ho Lee ${ }^{1}$, Jeong Hoon Kim ${ }^{2}$, Young Hee Yoon ${ }^{3}$, Hyun \\ Sook Chi ${ }^{4}$, Sang Wook Lee ${ }^{5}$, Chan-Sik Park ${ }^{6}$, Jooryung Huh ${ }^{6}$, Cheolwon Suh ${ }^{1}$ \\ Departments of ${ }^{1}$ Oncology, ${ }^{2}$ Neurological Surgery, ${ }^{3}$ Ophthalmology, ${ }^{4}$ Laboratory Medicine, ${ }^{5}$ Radiation Oncology and ${ }^{6}$ Pathology, \\ Asan Medical Center, University of Ulsan College of Medicine, Seoul, Korea
}

p-ISSN 1738-7949 / e-ISSN 2092-9129

http://dx.doi.org/10.5045/kjh.2012.47.1.60

Korean J Hematol 2012;47:60-6.

Received on February 17, 2012

Revised on March 13, 2012

Accepted on March 14, 2012

\author{
Correspondence to \\ Cheolwon Suh, M.D., Ph.D. \\ Department of Oncology, Asan Medical \\ Center, University of Ulsan College of \\ Medicine, 88 Olympic-ro 43-gil, \\ Songpa-gu, Seoul 138-736, Korea \\ Tel: $+82-2-3010-3209$ \\ Fax: +82-2-3010-6961 \\ E-mail: csuh@amc.seoul.kr \\ (C) 2012 Korean Society of Hematology
}

\section{Background}

Primary central nervous system lymphoma (PCNSL) rarely relapses in extracranial sites, and no specialized guidelines for follow-up evaluation have been proposed.

\section{Methods}

We analyzed 65 patients with newly diagnosed PNCSL to evaluate the pattern of relapse and prognostic factors.

\section{Results}

Of the 65 patients analyzed, 55 had only parenchymal brain disease, and 10 had both intracranial and extracranial lesions. As a first-line treatment, 29 patients received chemotherapy only (CTx), 13 received chemotherapy followed by whole brain radiotherapy (CTx-WBRT), 18 received chemotherapy followed by autologous stem cell transplantation (CTx-ASCT), 2 received palliative WBRT, and 3 received best supportive care. The overall response rate to the initial treatment was $75.8 \%$, with specific response rates of $62.1 \%$ to CTx, $84.6 \%$ to CTx-WBRT, and $100 \%$ to CTx-ASCT. The complete response (CR) rate was higher with CTx-ASCT than in the absence of ASCT (77.8\% vs. $43.2 \%$; $P=0.025)$. After a median follow-up of 18.8 months, the median failure-free survival (FFS) and overall survival (OS) were 13.0 and 36.1 months, respectively. No systemic relapse without a CNS lesion was noted. Multivariate analysis showed that ASCT was predictive of better FFS but not of OS. Age and the Memorial-Sloan Kettering Cancer Center prognostic score were predictive of survival.

\section{Conclusion}

We observed no systemic relapse without a CNS lesion, suggesting that regular systematic evaluation of extracranial sites may not always be necessary. Age was prognostic of survival irrespective of treatment scheme. ASCT may improve CR rate and FFS.

Key Words Primary CNS lymphoma, Relapse, Prognostic factor

\section{INTRODUCTION}

Primary central nervous system lymphoma (PCNSL) is a rare B-cell variant of non-Hodgkin lymphoma that is confined to the brain, leptomeninges, spinal cord, and eyes. Although PCNLS is sensitive to corticosteroids, chemotherapy, and radiotherapy, outcomes for patients with PCNSL are substantially worse than for patients at similar stages of systemic non-Hodgkin lymphoma. High-dose methotrexate (HD-MTX)-based chemotherapy or high-dose chemotherapy (HDC) followed by autologous stem cell trans- plantation (ASCT) are emerging as alternative strategies for patients with PCNSL [1-4]. Despite these advances in treatment, up to half of patients relapse after initial remission, and $10-15 \%$ of patients are primarily refractory to treatment [5]. PCNSL rarely relapses in extracranial sites, but a systematic approach to reevaluating disease status at relapse has not been utilized in these patients [5]. Furthermore, no specific guidelines have been proposed for follow-up evaluation of patients treated for PCNSL.

Prognostic models such as the International Extranodal Lymphoma Study group (IELSG) scoring system and the Memorial-Sloan Kettering Cancer Center (MSKCC) prog-

This is an Open Access article distributed under the terms of the Creative Commons Attribution Non-Commercial License (http://creativecommons.org/licenses/by-nc/3.0) which permits unrestricted non-commercial use, distribution, and reproduction in any medium, provided the original work is properly cited. 
nostic score based on age, performance status (PS), and extent of disease have been proposed to assess the prognosis of patients with PCNSL and to allow appropriate therapeutic decision making $[6,7]$. However, prognostic factors have not been assessed fully in patients with PCNSL treated with HD-MTX-based chemotherapy or HDC followed by ASCT. We therefore evaluated patterns of relapse and investigated prognostic factors for PCNSL in a single-center cohort.

\section{MATERIALS AND METHODS}

\section{Patients}

The study population included 65 patients newly diagnosed with PCNSL at Asan Medical Center, Seoul, Korea, between November 1995 and August 2010. Patient characteristics including age, Eastern Cooperative Oncology Group (ECOG) PS, international prognostic index (IPI), and treatment information were collected from the ASCT data registry at our center.

\section{Staging and response evaluation}

Staging evaluations for each patient involved a physical examination, including slit-lamp assessment by an ophthalmologist; contrast-enhanced magnetic resonance imaging (MRI) of the brain; computed tomography (CT) of the thorax, abdomen, and pelvis; lumbar puncture and cerebrospinal fluid (CSF) cytology; bilateral bone marrow (BM) aspiration and biopsy; serology test for HIV; complete blood cell count with differential count; liver and kidney function tests; and serum lactate dehydrogenase (LD) level measurements. Lumbar puncture was not performed in patients suspected of having increased intracranial pressure.

Responses to treatment were assessed according to the criteria of the International Group for PCNSL [8]. Routine follow-up imaging analysis (MRI of the brain and CT) and ophthalmologic exams were performed every 3 months or whenever clinically indicated.

\section{Statistical analysis}

Patient characteristics were described using summary statistics, as medians and ranges, or as proportions. Overall survival (OS) was calculated from the date of diagnosis, to the date of death from any cause or the date of last follow-up for surviving patients. Failure-free survival (FFS) was calculated from the date of diagnosis to date of relapse, progression of disease, death from any cause, or last follow-up. OS and FFS were estimated using the Kaplan-Meier method and compared using the log-rank test. Two-tailed $P$-values $<0.05$ were regarded as statistically significant.

\section{IELSG and MSKCC prognostic score}

The IELSG prognostic scoring system is composed of 5 prognostic variables: age more than 60 years, ECOG score more than 1 (Karnofsky performance status [KPS] $<70 \%$ ), elevated serum LD level, high CSF protein concentration, and involvement of the deep regions of the brain (periventric- ular regions, basal ganglia, brainstem, and/or cerebellum) [7]. Due to the retrospective nature of this study, resulting in incomplete data, we assessed only the prognostic significance of patient age, ECOG PS, and serum LD level.

The MSKCC prognostic score is a simplified index, including age and KPS [6], with 3 distinct prognostic classes: class 1 (age $<50$ years), class 2 (age $\geq 50$ years or KPS $\geq 70 \%$ ), and class 3 (age $\geq 50$ years and KPS $<70 \%$ ). This scoring system was also used for prognostic analysis.

\section{RESULTS}

\section{Patients}

The clinical characteristics of the 65 patients are summarized in Table 1. Their median age was 55 years (range, 26-77 years). Of these, 63 tumors were histologically diagnosed

Table 1. Baseline characteristics $(\mathrm{N}=65)$.

Age Median (Range)

Pathologic diagnosis

Diffuse large B-cell lymphoma $\quad 63(96.9 \%)$

T-cell lymphoma

$2(3.1 \%)$

Age

$\geq 60$

$<60$

$46(70.8 \%)$

$19(29.2 \%)$

Gender

Male

$43(66.2 \%)$

Female

$22(33.8 \%)$

Involvement sites

Intracranial lesion only

Extracranial involvement Leptomeningeal seeding

Ocular lesion

Spinal cord

$55(84.6 \%)$

$10(15.4 \%)$

7

B Symptom

Yes

No

$(1.5 \%)$

$64(98.5 \%)$

ECOG PS

1

$49(75.4 \%)$

$10(15.4 \%)$

$\begin{array}{ll}3 & 3(4.6 \%) \\ 4 & 3(4.6 \%)\end{array}$

IPI at diagnosis

0

$16(24.6 \%)$

$26(40.0 \%)$

$10(15.4 \%)$

$12(18.5 \%)$

$1(1.5 \%)$

1st line therapy

Chemotherapy alone

Chemotherapy followed by WBRT

$29(44.6 \%)$

$13(20.0 \%)$

Chemotherapy followed by ASCT

Palliative WBRT only ${ }^{\text {b }}$

$18(27.7 \%)$

$2(3.1 \%)$

Best supportive care

$3(4.6 \%)$

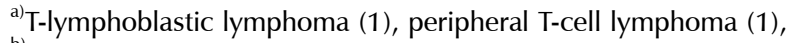
${ }^{b)}$ All were HIV-associated lymphoma.

Abbreviations: ECOG PS, Eastern Cooperative Oncology Group performance status; IPI, international prognostic index; WBRT, whole brain radiotherapy; ASCT, autologous stem cell transplantation. 
as diffuse large B-cell lymphoma and 2 as T-cell lymphomas (T-lymphoblastic lymphoma and peripheral T-cell lymphoma). All patients had parenchymal brain disease, and 10 patients (15.4\%) had extracranial involvement including 7 with positive CSF cytology, 2 with ocular involvement, and 1 with a spinal cord lesion. Twenty-nine (44.3\%) patients received chemotherapy alone $(\mathrm{CTx})$ as first-line treatment, while $13(20.0 \%)$ received CTx followed by whole brain radiotherapy (WBRT) and 18 (27.7\%) received CTx followed by ASCT. Two patients with HIV infection received only palliative WBRT, and 3 received best supportive care without any chemotherapy or radiation therapy because of poor PS. No difference in patient characteristics was observed between those treated with and without ASCT (data not shown).

\section{Response and survival after treatment}

As shown in Table 2, 62 of the 65 patients received first-line treatment with curative intent. The overall response rates (ORR) in patients receiving CTx alone, and CTx followed by WBRT, were $62.1 \%$ and $84.6 \%$, respectively. All patients who received chemotherapy followed by ASCT achieved clinical response; of these $77.8 \%$ showed complete response
(CR) and 12.2\% showed partial response (PR). Among the 18 patients treated with CTx followed by ASCT, 5 patients showed PR after CTx, but finally achieved CR after ASCT. The ORR $(P=0.031)$ and $\mathrm{CR}$ rate $(P=0.025)$ were significantly higher in patients treated with chemotherapy followed by ASCT than in those treated without ASCT (Table 2).

After a median follow-up duration of 18.8 months (range, 1.1-138.0 months), 31 of the 65 patients had died including 21 from progression or relapse of PCNSL. The median FFS was 13.0 months (95\% confidence interval [CI], 3.5-22.8 months) and the median OS was 36.1 months (95\% CI, 20.5-51.7 months). The 2-year FFS and OS rates were $42.0 \%$ and $66.5 \%$, respectively (Fig. 1). Four treatment-related deaths occurred in patients who received HD-MTX chemotherapy.

\section{Failure of disease and salvage treatment}

The 3 patients who received best supportive care without other treatment showed progression of intracranial lesions, with 1 patient dying from severe pneumonia. Following first-line treatment, 6 patients $(9.2 \%)$ experienced progression of disease, and 25 (38.5\%) experienced relapse of disease, including 21 with brain parenchymal lesions, 1 with

Table 2. Responses to first-line treatment $(\mathrm{N}=62)$.

\begin{tabular}{|c|c|c|c|c|c|c|}
\hline \multirow{2}{*}{ Response } & \multicolumn{4}{|c|}{ Non-ASCT $(\mathrm{N}=44)$} & \multirow{2}{*}{$\begin{array}{c}\text { CTx-ASCT } \\
(\mathrm{N}=18)\end{array}$} & \multirow{2}{*}{$P^{\mathrm{a})}$} \\
\hline & $\mathrm{CTx}(\mathrm{N}=29)$ & CTx-WBRT $(\mathrm{N}=13)$ & Palliative WBRT only $(\mathrm{N}=2)$ & All & & \\
\hline CR & 13 (44.9\%) & 6 (46.1\%) & $0(0.0 \%)$ & $19(43.2 \%)$ & $14(77.8 \%)$ & 0.025 \\
\hline PR & $5(17.2 \%)$ & 5 (38.5\%) & $0(0.0 \%)$ & $10(22.8 \%)$ & $4(12.2 \%)$ & \\
\hline ORR & $62.1 \%$ & $84.6 \%$ & & $66.0 \%$ & $90.0 \%$ & 0.031 \\
\hline SD & $2(6.9 \%)$ & $1(7.7 \%)$ & $0(0.0 \%)$ & $3(6.8 \%)$ & $0(0.0 \%)$ & \\
\hline PD & 3 (10.3\%) & $1(7.7 \%)$ & $2(100.0 \%)$ & $6(13.6 \%)$ & $0(0.0 \%)$ & \\
\hline $\mathrm{NE}$ & $6(20.7 \%)$ & $0(0.0 \%)$ & $0(0.0 \%)$ & $6(13.6 \%)$ & $0(0.0 \%)$ & \\
\hline
\end{tabular}

${ }^{a)} P$-value indicates comparison between non-ASCT and CTx-ASCT groups.

Abbreviations: $C R$, complete response; $P R$, partial response; $O R R$, overall response rate $(C R+P R)$; $S D$, stable disease; $P D$, progressive disease; $\mathrm{NE}$, not evaluable; CTx, chemotherapy; WBRT, whole brain radiotherapy; ASCT, autologous stem cell transplantation.
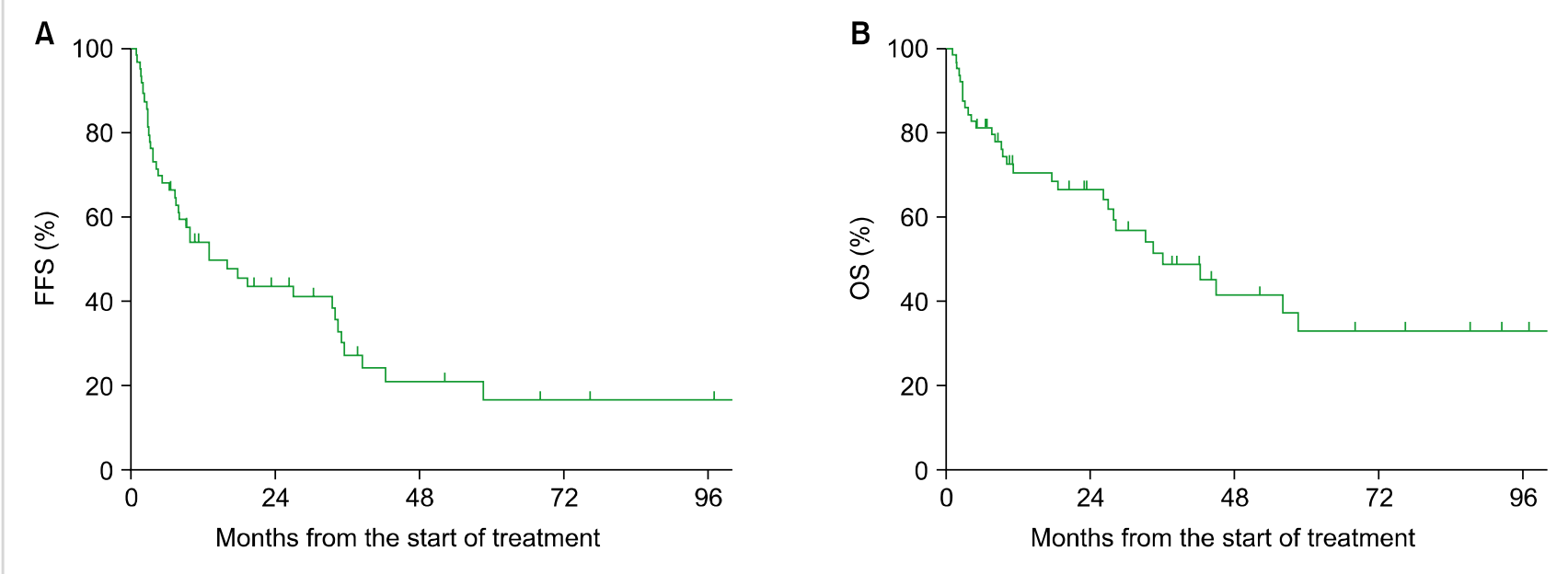

Fig. 1. Failure free survival (A) and overall survival (B) in all patients. 
an ocular lesion, and 3 with positive CSF cytology (Table 3). No systemic relapse was observed without a CNS lesion.

Following failure of first-line treatment, 33 patients received salvage treatment, including 15 who received salvage CTx, 4 who received salvage CTx followed by ASCT, 11 who received WBRT, and 3 who received best supportive care alone.

\section{Analysis for prognostic factors}

FFS was not related to patient age, ECOG PS, serum LD level, or MSKCC prognostic score. Age less than 60 years $(P=0.001)$, ECOG PS $\leq 1 \quad(P=0.019)$, and lower MSKCC prognostic score $(P=0.001)$ were related to prolonged OS (Table 4 and Fig. 2). However, patients who received HDC followed by ASCT showed significantly better FFS (58.6 months vs. 9.1 months; $P=0.006)$ and OS (58.6 months vs. 33.3 months; $P=0.034$ ) than those who did not (Fig. 3).

\begin{tabular}{lc} 
Table 3. Failure pattern of disease $(\mathrm{N}=65)$. \\
\hline Progression after best supportive care & $2(3.0 \%)$ \\
Intracranial lesion & 2 \\
Progression after first line treatment ${ }^{\mathrm{a})}$ & $6(9.2 \%)$ \\
Intracranial lesion $_{\text {Extracranial lesion }}$ & 5 \\
Leptomeningeal seeding & 1 \\
Relapse after first line treatment ${ }^{\text {b) }}$ & 1 \\
Intracranial lesion & $25(38.5 \%)$ \\
Extracranial lesion & 21 \\
Ocular lesion & 4 \\
Leptomeningeal seeding & 1 \\
No failure of disease & 3 \\
Unknown & $25(38.5 \%)$ \\
\end{tabular}

${ }^{a}$ Chemotherapy only (4), Whole brain radiotherapy [WBRT] (3),

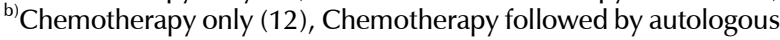
stem cell transplantation (8), Chemotherapy followed by WBRT (7).
Multivariate analysis showed that ASCT was an independent predictive factor for better FFS (hazard ratio [HR], 0.348; 95\% CI, 0.151-0.084), but not for OS (Table 5). Age equal to or more than 60 years was the only independent prognostic factor for OS (HR, 2.968; 95\% CI, 1.269-6.939). The MSKCC prognostic score was also predictive of patient survival in multivariate analysis.

\section{DISCUSSION}

All of the patients with PCNSL in this study were systematically evaluated at the initial staging and at follow-up, by methods including CTs of the thorax, abdomen and pelvis, lumbar puncture, and positron emission tomography (PET). Analysis of the relapse pattern of PCNSL showed that no patient experienced a systemic relapse without a CNS lesion, in accordance with earlier studies showing that most patients with PCNSL relapsed in the CNS, whereas systemic relapse and simultaneous CNS and systemic relapse were infrequent [5, 9-12]. These studies, however, may have underestimated the frequency of relapse without a CNS lesion, due to the lack of systematic follow-up staging evaluation.

In one of the largest series evaluating the characteristics of patients with relapsed PCNSL, only 6 of 143 patients had isolated systemic relapses [5]. However, not all patients underwent systematic reevaluation of disease status, including CSF analysis and eye examinations, at relapse. In another study, 10 of 209 patients (4.8\%) experienced extracerebral relapse without CNS progression [13]. The data of patients in that study, however, was collected retrospectively from several databases, suggesting that systematic evaluation of disease progression might not have been performed using the same methods. In accordance with the findings of these previous studies, we found that isolated systemic relapse without a CNS lesion was extremely rare, even with regular

Table 4. Univariate analysis for overall survival and failure-free survival.

\begin{tabular}{|c|c|c|c|c|}
\hline & FFS, median $(95 \% \mathrm{Cl})$ & $P$ & OS, median $(95 \% \mathrm{Cl})$ & $P$ \\
\hline Age & & 0.430 & & 0.001 \\
\hline$<60$ years & $16.0 \pm 15.4(0.0-46.2)$ & & $58.6 \pm 11.5(56.2-101.4)$ & \\
\hline$\geq 60$ years & $9.9 \pm 5.1(0.0-20.0)$ & & $18.7 \pm 8.7(1.6-35.7)$ & \\
\hline ECOG PS & & 0.219 & & 0.019 \\
\hline$\leq 1$ & $13.1 \pm 5.3(2.8-23.5)$ & & $56.0 \pm 13.1(30.3-81.8)$ & \\
\hline$>1$ & $9.1 \pm 2.4(4.5-13.7)$ & & $26.1 \pm 13.1(0.4-51.7)$ & \\
\hline LD & & 0.883 & & 0.554 \\
\hline Normal level & $9.9 \pm 3.3(3.4-16.3)$ & & $56.20 \pm 24.0(8.9-103.1)$ & \\
\hline$>$ Normal level & $16.0 \pm 10.7(0.0-37.1)$ & & $34.6 \pm 8.6(17.7-51.5)$ & \\
\hline MSKCC group & & 0.328 & & 0.001 \\
\hline Age $\leq 50$ years & $9.9 \pm 5.0(0.1-19.6)$ & & Not reached & \\
\hline Age $>50$ years and $K P S \geq 70 \%$ & $19.4 \pm 13.1(0.0-45.0)$ & & $42.4 \pm 12.5(18.0-66.8)$ & \\
\hline Age $>50$ years and $\mathrm{KPS}<70 \%$ & $8.0 \pm 1.4(5.2-10.7)$ & & $11.3 \pm 13.8(0.0-38.5)$ & \\
\hline ASCT & & 0.006 & & 0.034 \\
\hline Done & $58.6 \pm 22.3(14.9-102.4)$ & & $58.6 \pm 7.1(43.9-71.9)$ & \\
\hline Not done & $9.1 \pm 4.4(0.4-17.8)$ & & $33.3 \pm 6.7(20.2-46.5)$ & \\
\hline
\end{tabular}

Abbreviations: ECOG PS, Eastern Cooperative Oncology Group performance status; KPS, Karnofsky performance status; ASCT, autologous stem cell transplantation; FFS, failure-free survival; OS, overall survival; MSKCC, Memorial-Sloan Kettering Cancer Center. 

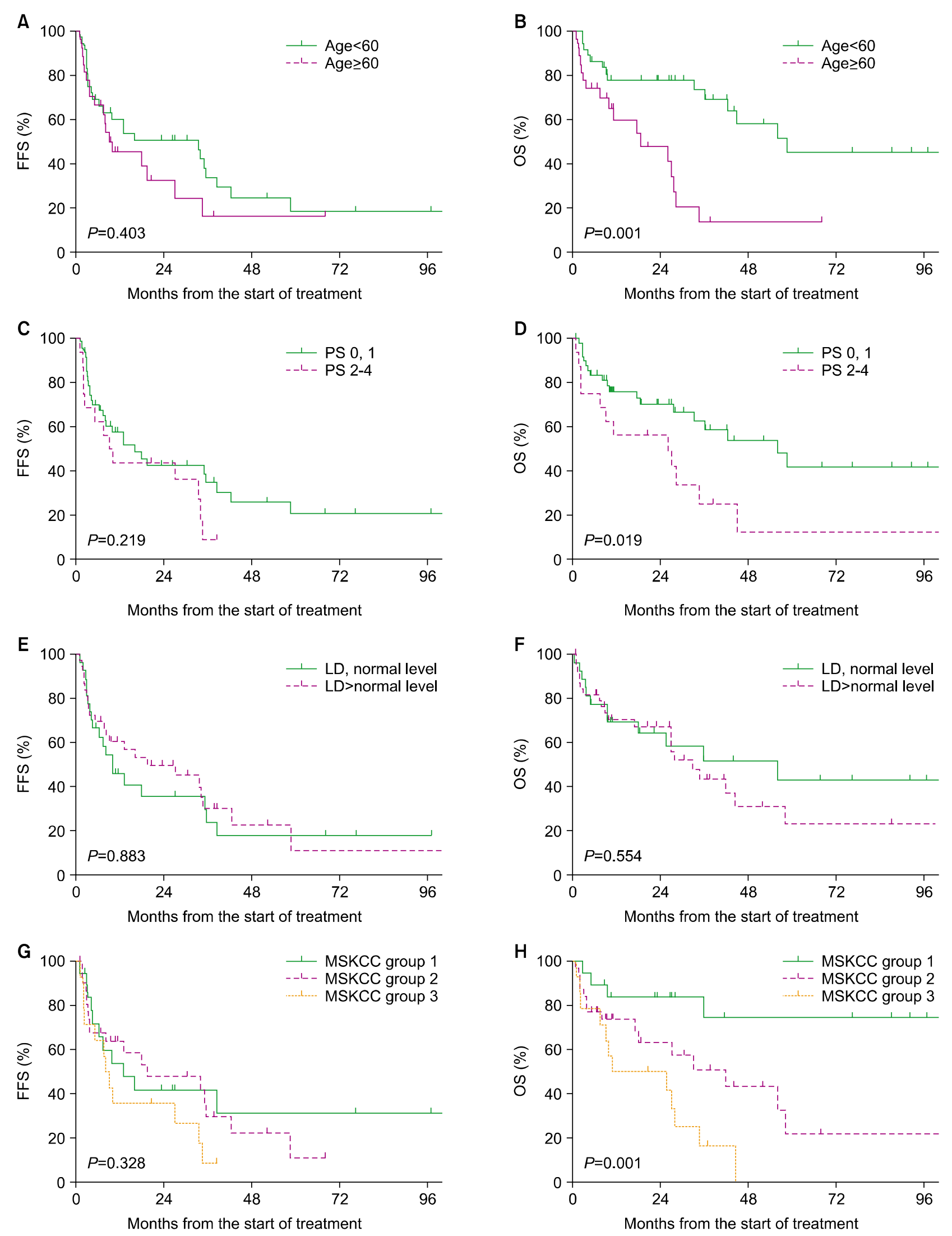

Fig. 2. Failure free survival and overall survival according to age (A, B), Eastern Cooperative Oncology Group performance status (ECOG PS) (C, D), serum lactate dehydrogenase (LD) level (E, F), and MSKCC, Memorial-Sloan Kettering Cancer Center (MSKCC) prognostic group (G, H). 

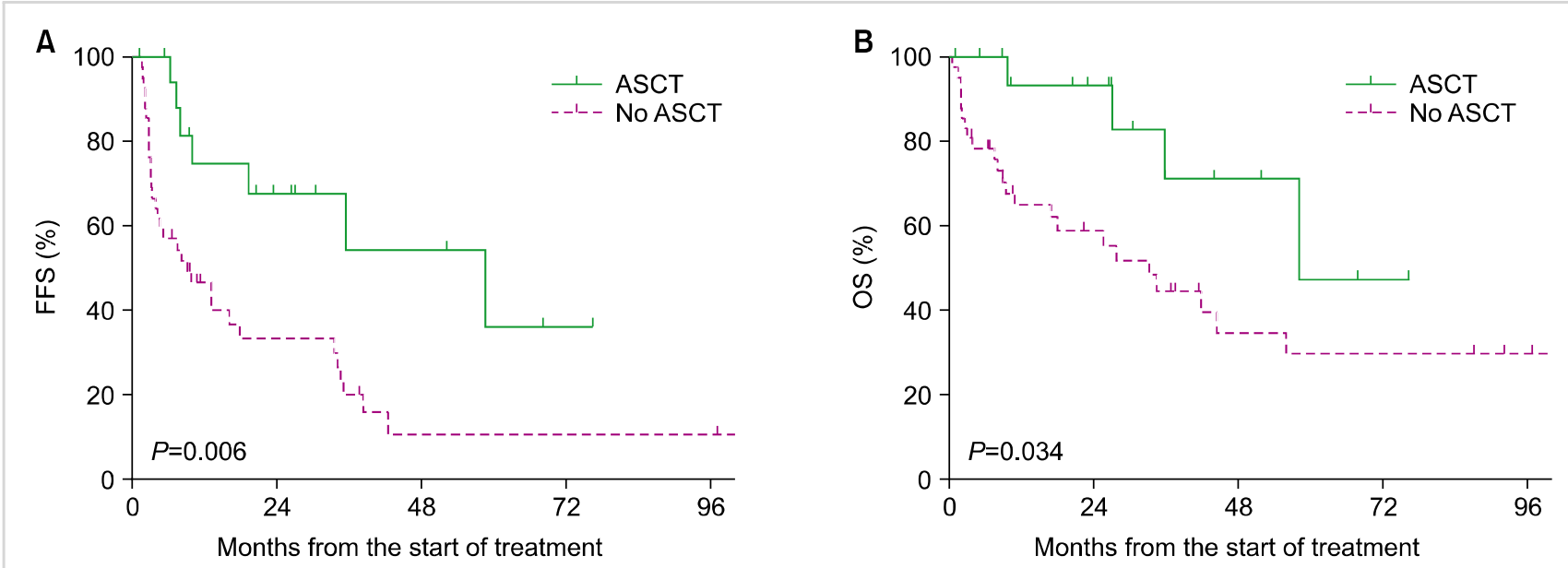

Fig. 3. Failure free survival (A) and overall survival (B) in patients treated with and without autologous stem cell transplantation.

Table 5. Multivariate analysis for overall survival and failure-free survival.

\begin{tabular}{|c|c|c|c|c|c|c|}
\hline & & FFS & & & OS & \\
\hline & $\mathrm{HR}$ & $95 \% \mathrm{Cl}$ & $P$ & $\mathrm{HR}$ & $95 \% \mathrm{Cl}$ & $P$ \\
\hline ASCT & 0.348 & $0.151-0.804$ & 0.013 & 0.384 & $0.129-1.141$ & 0.085 \\
\hline Age $\geq 60$ & 1.100 & $0.557-2.171$ & 0.784 & 2.968 & $1.269-6.939$ & 0.012 \\
\hline ECOG PS > 1 & 1.283 & $0.619-2.656$ & 0.503 & 1.529 & $0.670-3.489$ & 0.313 \\
\hline ASCT & 0.348 & $0.151-0.802$ & 0.013 & 0.370 & & 0.073 \\
\hline MSKCC group & & & & & & \\
\hline 2 & 0.651 & $0.268-1.585$ & 0.344 & 4.364 & $1.683-11.310$ & 0.002 \\
\hline 3 & 0.724 & $0.330-1.591$ & 0.422 & 2.676 & $1.178-6.079$ & 0.019 \\
\hline
\end{tabular}

Abbreviations: FFS, failure-free survival; OS, overall survival; ECOG PS, Eastern Cooperative Oncology Group performance status; MSKCC, Memorial-Sloan Kettering Cancer Center group.

1: Age $\leq 50$ years. 2: Age $>50$ years and $\mathrm{KPS} \geq 70 \%$. 3: Age $>50$ years and $\mathrm{KPS}<70 \%$.

systematic evaluation by brain MRI, CTs of chest, abdomen, and pelvis, and eye examinations, as none of our patients showed isolated systemic relapse. These findings suggest that complete systematic evaluation may be unnecessary, and brain imaging alone may be sufficient for follow-up of patients with PCNSL.

The IELSG prognostic score model is not readily applicable in patients with PCNSL, due to its complexity [7], whereas the MSKCC prognostic score, based on age and PS, is simpler and has higher applicability [6]. Data on prognostic factors are limited in patients treated with HD-MTX-based chemotherapy or HDC followed by ASCT. We therefore analyzed several prognostic factors in 65 patients with PCNSL, including 18 who had been treated with HD-MTX-based chemotherapy followed by ASCT. In accordance with the IELSG and MSKCC prognostic models, we found that age and PS were associated with OS irrespective of treatment. However, due to a lack of information regarding CSF protein levels and the depth of brain lesions in some patients, the IELSG prognostic score was not applicable in this population. In contrast, the MSKCC prognostic score was useful in predicting OS in our patients.
HD-MTX-based chemotherapy followed by ASCT may improve outcomes in selected patients with PCNSL $[2,4]$. ASCT may significantly improve both the CR rate and FFS. However, due to the retrospective nature of this study and the small number of patients, our findings require validation in other, larger retrospective and prospective trials.

In conclusion, the observed absence of systemic involvement without a CNS lesion in patients with relapsed PCNSL suggests that regular systematic evaluation to investigate extracranial relapse may not be always necessary in patients with PCNSL. Age was a prognostic factor for OS, irrespective of treatment scheme, and ASCT may improve the CR rate and FFS.

\section{REFERENCES}

1. Milpied N, Deconinck E, Gaillard F, et al. Initial treatment of aggressive lymphoma with high-dose chemotherapy and autologous stem-cell support. N Engl J Med 2004;350:1287-95.

2. Ferreri AJ, Crocchiolo R, Assanelli A, Govi S, Reni M. High-dose chemotherapy supported by autologous stem cell transplantation 
in patients with primary central nervous system lymphoma: facts and opinions. Leuk Lymphoma 2008;49:2042-7.

3. Morris PG, Abrey LE. Therapeutic challenges in primary CNS lymphoma. Lancet Neurol 2009;8:581-92.

4. Yoon DH, Lee DH, Choi DR, et al. Feasibility of BU, CY and etoposide (BUCYE), and auto-SCT in patients with newly diagnosed primary CNS lymphoma: a single-center experience. Bone Marrow Transplant 2011;46:105-9.

5. Jahnke K, Thiel E, Martus P, et al. Relapse of primary central nervous system lymphoma: clinical features, outcome and prognostic factors. J Neurooncol 2006;80:159-65.

6. Abrey LE, Ben-Porat L, Panageas KS, et al. Primary central nervous system lymphoma: the Memorial Sloan-Kettering Cancer Center prognostic model. J Clin Oncol 2006;24:5711-5.

7. Ferreri AJ, Blay JY, Reni M, et al. Prognostic scoring system for primary CNS lymphomas: the International Extranodal Lymphoma Study Group experience. J Clin Oncol 2003;21:266-72.

8. Abrey LE, Batchelor TT, Ferreri AJ, et al. Report of an international workshop to standardize baseline evaluation and response criteria for primary CNS lymphoma. J Clin Oncol 2005;23:5034-43.
9. Blay JY, Conroy T, Chevreau C, et al. High-dose methotrexate for the treatment of primary cerebral lymphomas: analysis of survival and late neurologic toxicity in a retrospective series. J Clin Oncol 1998;16:864-71.

10. Abrey LE, Moskowitz CH, Mason WP, et al. Intensive methotrexate and cytarabine followed by high-dose chemotherapy with autologous stem-cell rescue in patients with newly diagnosed primary CNS lymphoma: an intent-to-treat analysis. J Clin Oncol 2003;21:4151-6.

11. Batchelor T, Carson K, O'Neill A, et al. Treatment of primary CNS lymphoma with methotrexate and deferred radiotherapy: a report of NABTT 96-07. J Clin Oncol 2003;21:1044-9.

12. DeAngelis LM, Seiferheld W, Schold SC, Fisher B, Schultz CJ. Combination chemotherapy and radiotherapy for primary central nervous system lymphoma: Radiation Therapy Oncology Group Study 93-10. J Clin Oncol 2002;20:4643-8.

13. Provencher S, Ferlay C, Alaoui-Slimani K, et al. Clinical characteristics and outcome of isolated extracerebral relapses of primary central nervous system lymphoma: a case series. Hematol Oncol 2011;29:10-6. 